\title{
AN EXISTENCE ANALYSIS FOR NONLINEAR EQUATIONS IN HILBERT SPACE $\left({ }^{1}\right)$
}

\author{
BY \\ JOHN LOCKER
}

1. Introduction. In this paper we present an existence analysis for the equation $L x=N x$, where $L$ is an unbounded linear operator in a Hilbert space $S$, and $N$ is a nonlinear operator in $S$. The conditions imposed on $L$ are satisfied by ordinary differential operators, and consequently, the theory is applicable to existence problems of nonlinear ordinary differential equations.

The theory reduces the existence problem to the study of a finite system of equations in finitely many unknowns. This technique has had widespread application to nonlinear analysis, beginning with Poincaré [11] in his work on celestial mechanics. It has been used by Schmidt [12] and Ljapunov [9] in their work on nonlinear integral equations, and by Bartle [2], Cronin [6], and Nirenberg [10], who extended their results. The latter study an equation similar to ours, assuming $L$ to be a bounded everywhere-defined operator in a Banach space.

Cesari [3] has recently developed an existence theory for the equation $L x=N x$ in a Hilbert space $S$. He presents a system of axioms for the existence of linear operators $H$ and $P$. Using these operators, he also reduces the existence problem to solving a finite system of equations in finitely many unknowns.

The theory of this paper is closely related to Cesari's theory, coinciding with his if $L$ is a selfadjoint ordinary differential operator. Let $S$ be a real Hilbert space with inner product $(x, y)$ and norm $\|x\|$. The symbols $\mathscr{D}(L)$ and $\mathscr{R}(L)$ denote the domain and range, respectively, of any operator $L$ defined in $S$. If $L$ is linear, $\mathscr{N}(L)$ denotes the null space of $L$ and $L^{*}$ denotes the adjoint of $L$ in case $\mathscr{D}(L)$ is dense in $S$.

Let $L$ be a closed linear operator in $S$ with the following properties:

(Ia) $\mathscr{D}(L)$ is dense in $S$,

(Ib) $\mathscr{R}(L)$ is closed in $S$,

(Ic) $\operatorname{dim} \mathscr{N}(L)=p<\infty$ and $\operatorname{dim} \mathscr{N}\left(L^{*}\right)=q<\infty$.

Let $N$ be an operator in $S$ with $\mathscr{D}(L) \cap \mathscr{D}(N) \neq \varnothing$, and consider the equation

$$
L x=N x \text {. }
$$

Under the assumptions on $L$ we show that there exist linear operators $H, P$, and $Q$ with properties analogous to the properties of Cesari's operators $H$ and $P$.

Received by the editors January 28, 1966, and in revised form, October 28, 1966.

(1) The results in this paper are a part of the author's doctoral dissertation, which was written under the direction of Professor Lamberto Cesari at the University of Michigan. 
Utilizing these operators, we establish the existence of at least one solution $\hat{x} \in S$ to equation (1) provided a set of inequalities (which relate $L$ and $N$ ) can be satisfied and provided a finite system of equations in finitely many unknowns is solvable (see Theorem 3), and also obtain estimates on the norm of such a solution $\hat{x}$. For the case when the system of equations has either more or the same number of unknowns as equations, we present two existence theorems (see Theorem 4 and Theorem 5).

To conclude this paper we illustrate our theory by studying the nonlinear boundary value problem:

$$
\begin{aligned}
x^{\prime \prime}(t)+x(t)+\alpha x^{2}(t) & =\beta t, \quad 0 \leqq t \leqq 2 \pi, \\
x(0) & =0,
\end{aligned}
$$

where $\alpha$ and $\beta$ are constants. We show that this equation has a solution if $|\alpha| \leqq 1$, $|\beta| \leqq .001$, and obtain estimates on the norm of such a solution.

In another paper we shall examine our existence theory when $L$ is an ordinary differential operator on a finite interval $[a, b]$ and $S$ is the Hilbert space $L_{2}[a, b]$. For this case the theory assumes a specialized form which is convenient for practical applications to nonlinear ordinary differential equations.

2. The existence analysis. Choose elements $\phi_{1}, \ldots, \phi_{p}$ in $\mathscr{D}(L)$ to form an orthonormal base for $\mathscr{N}(L)$; choose elements $\omega_{1}, \ldots, \omega_{q}$ in $\mathscr{D}\left(L^{*}\right)$ to form an orthonormal base for $\mathscr{N}\left(L^{*}\right)$. Letting $\mathscr{N}(L)^{\perp}$ denote the orthogonal complement of $\mathscr{N}(L)$ in $S$, we note that the restriction of $L$ to $\mathscr{D}(L) \cap \mathscr{N}(L)^{\perp}$ is a 1-1 closed operator having the same range as $L$. Let $H$ denote the inverse of this operator:

$$
H=\left[L \mid \mathscr{D}(L) \cap \mathscr{N}(L)^{\perp}\right]^{-1} .
$$

By the Closed Graph Theorem $H$ is a 1-1 continuous linear operator, and clearly $\mathscr{D}(H)=\mathscr{R}(L), \mathscr{R}(H)=\mathscr{D}(L) \cap \mathscr{N}(L)^{\perp}$, and

$$
\begin{aligned}
& L H y=y \quad \text { for all } y \in \mathscr{R}(L), \\
& H L x=x-\sum_{i=1}^{p}\left(x, \phi_{i}\right) \phi_{i} \quad \text { for all } x \in \mathscr{D}(L) .
\end{aligned}
$$

Thus, $H$ is a continuous right inverse for $L$. It plays a major role in our existence theory.

Let $m$ be an integer with $m \geqq q$, and choose elements $\omega_{q+1}, \ldots, \omega_{m}$ in $\mathscr{D}\left(L^{*}\right)$ such that the elements $\omega_{1}, \ldots, \omega_{m}$ form an orthonormal set in $S$. Since $S$ is the orthogonal direct sum of $\mathscr{N}\left(L^{*}\right)$ and $\mathscr{R}(L)$, the elements $\omega_{q+1}, \ldots, \omega_{m}$ belong to $\mathscr{R}(L)$, and hence, the elements $H \omega_{q+1}, \ldots, H \omega_{m} \in \mathscr{D}(L) \cap \mathscr{N}(L)^{\perp}$ can be formed.

Let $S_{0}$ be the subspace spanned by the elements $\phi_{1}, \ldots, \phi_{p}$, and $H \omega_{q+1}, \ldots, H \omega_{m}$. Since these elements are linearly independent, $S_{0}$ has dimension $p+m-q$. Henceforth, we assume that $S_{0}$ is a subset of $\mathscr{D}(N)$, which implies that $S_{0}$ is a subset of $\mathscr{D}(L) \cap \mathscr{D}(N)$. 
Let $P$ and $Q$ be the projection operators defined in $S$ by

$$
P x=\sum_{i=1}^{m}\left(x, \omega_{i}\right) \omega_{i} \text { for all } x \in S,
$$

and

$$
Q x=\sum_{i=1}^{p}\left(x, \phi_{i}\right) \phi_{i}+\sum_{i=q+1}^{m}\left(x, L^{*} \omega_{i}\right) H \omega_{i} \text { for all } x \in S .
$$

These two operators have the following properties: (a) they are continuous linear operators defined on all of $S$, (b) $\mathscr{R}(P)$ is the subspace $\left\langle\omega_{1}, \ldots, \omega_{m}\right\rangle$ spanned by $\omega_{1}, \ldots, \omega_{m}$, and $\mathscr{R}(P) \subseteq \mathscr{D}\left(L^{*}\right)$, (c) $\mathscr{R}(Q)=S_{0} \subseteq \mathscr{D}(L)$, and (d) $P^{2}=P, Q^{2}=Q$. Also, the range of $I-P$ is a subset of $\mathscr{R}(L)$, and hence, $H(I-P)$ is a continuous linear operator defined on all of $S$.

THEOREM 1. The following properties are valid:

(a) $H(I-P) L x=(I-Q) x$ for all $x \in \mathscr{D}(L)$.

(b) $L H(I-P) x=(I-P) x$ for all $x \in S$.

(c) $L Q x=P L x$ for all $x \in \mathscr{D}(L)$.

(d) $Q H(I-P) x=0$ for all $x \in S$.

Proof. To show (a), take $x \in \mathscr{D}(L)$. Then

$$
\begin{aligned}
(I-P) L x & =L x-\sum_{i=1}^{m}\left(L x, \omega_{i}\right) \omega_{i} \\
& =L x-\sum_{i=q+1}^{m}\left(x, L^{*} \omega_{i}\right) \omega_{i},
\end{aligned}
$$

and hence, by (4) we have

$$
\begin{aligned}
H(I-P) L x & =H L x-\sum_{i=q+1}^{m}\left(x, L^{*} \omega_{i}\right) H \omega_{i} \\
& =(I-Q) x .
\end{aligned}
$$

(b) follows from (3), while (c) and (d) can be shown by direct computation.

The properties listed in Theorem 1 are analogous to the properties satisfied by Cesari's operators in [3]. We use these properties to develop an existence theory for equation (1).

Suppose $x \in \mathscr{D}(L) \cap \mathscr{D}(N)$ with $L x=N x$. By Theorem 1(a) we have

$$
x=x^{*}+H(I-P) N x
$$

where $x^{*}=Q x \in S_{0}$. Let us try to reverse this argument. Take $x^{*} \in S_{0}$ and suppose there exists $x \in \mathscr{D}(N)$ satisfying (7). Clearly $x \in \mathscr{D}(L)$, and by Theorem $1(\mathrm{~d})$ we have $Q x=Q x^{*}=x^{*}$. Thus, $L x=L Q x+L H(I-P) N x$. Using parts (b) and (c) of Theorem 1, we get

$$
L x-N x=P(L x-N x) .
$$

Therefore, $x$ is a solution of (1) if and only if

$$
P(L x-N x)=0 \text {. }
$$


We have shown that if $x \in \mathscr{D}(N)$ is a solution of equation (7) corresponding to $x^{*} \in S_{0}$ and if $x$ is a solution of equation (9), then $x$ is a solution of the original equation (1). Equation (7) is called the auxiliary equation.

In the next section we introduce sufficient conditions for the existence of a unique solution $x$ to the auxiliary equation (7) corresponding to each $x^{*}$ belonging to a subset $V$ of $S_{0}$. Then in the following section we establish sufficient conditions that there exist $x^{*} \in V$ such that the corresponding element $x$ also satisfies equation (9), and hence, yields a solution to equation (1).

3. The auxiliary equation. Let $S^{\prime}$ be a subspace in $S$ and let $\mu$ be a seminorm defined in $S^{\prime}$. We assume that the following condition is satisfied:

(IIa) $\mathscr{D}(L)$ is a subset of $S^{\prime}$.

In applications to ordinary differential equations $S$ is the Hilbert space $L_{2}[a, b]$, $L$ is a differential operator in $S$ whose domain $\mathscr{D}(L)$ consists of functions which are at least continuous, $S^{\prime}$ is the set of functions in $S$ which are bounded almost everywhere, and $\mu$ is the uniform norm on $S^{\prime}$. For this case condition (IIa) is certainly satisfied.

We assume that the following condition is satisfied:

(IIb) There exist constants $k \geqq 0$ and $k^{\prime} \geqq 0$ such that

$$
\|H(I-P) x\| \leqq k\|x\|, \quad \mu(H(I-P) x) \leqq k^{\prime}\|x\| \quad \text { for all } x \in S .
$$

Choose an element $x_{0} \in S_{0}$. Noting that $x_{0} \in \mathscr{D}(L) \cap \mathscr{D}(N)$, let $\gamma=H(I-P) N x_{0}$. Choose constants $e$ and $e^{\prime}$ such that $\|\gamma\| \leqq e, \mu(\gamma) \leqq e^{\prime}$. Let $c, d, r$, and $R_{0}$ be real numbers with $0<c<d$ and $0<r<R_{0}$, and define sets $V$ and $\widetilde{S}_{0}$ in $S$ by

$$
V=\left\{x \in S_{0} \mid\left\|x-x_{0}\right\| \leqq c, \mu\left(x-x_{0}\right) \leqq r\right\}
$$

and

$$
\tilde{S}_{0}=\left\{x \in S^{\prime} \mid\left\|x-x_{0}\right\| \leqq d, \mu\left(x-x_{0}\right) \leqq R_{0}\right\} .
$$

Clearly $x_{0} \in V \subseteq \tilde{S}_{0}$, so these sets are nonempty. For each $x^{*} \in V$ let

$$
S\left(x^{*}\right)=\left\{x \in S^{\prime} \mid Q x=x^{*},\left\|x-x_{0}\right\| \leqq d, \mu\left(x-x_{0}\right) \leqq R_{0}\right\} .
$$

Clearly $x^{*} \in S\left(x^{*}\right)$, so each of the sets $S\left(x^{*}\right)$ is nonempty, and $x^{*} \in S\left(x^{*}\right) \subseteq \widetilde{S}_{0}$ for all $x^{*} \in V$.

Finally, we assume the following two conditions are satisfied:

(IIc) The set $\widetilde{S}_{0}$ is a subset of $\mathscr{D}(N)$, and there exists a constant $l \geqq 0$ such that

$$
\|N x-N y\| \leqq l\|x-y\| \text { for all } x, y \in \tilde{S}_{0} .
$$

(IId) For each $x^{*} \in V$ the set $S\left(x^{*}\right)$ is closed in $S$.

THEOREM 2. If conditions (Iabc) and (IIabcd) are satisfied and if

$$
k l<1, \quad c+e \leqq(1-k l) d, \quad r+e^{\prime} \leqq R_{0}-k^{\prime} l d,
$$

then for each $x^{*} \in V$ there exists a unique element $x \in S\left(x^{*}\right)$ which is a solution to the auxiliary equation (7) corresponding to $x^{*}$. Furthermore, $x \in \mathscr{D}(L) \cap \mathscr{D}(N)$, $Q x=x^{*}$, and $L x-N x=P(L x-N x)$. Also, the solutions $x$ vary continuously with the $x^{*}$. 
Proof. Fix $x^{*} \in V$, and let $T: S\left(x^{*}\right) \rightarrow S^{\prime}$ be the operator defined by

$$
T x=x^{*}+H(I-P) N x \text { for all } x \in S\left(x^{*}\right) \text {. }
$$

If $x \in S\left(x^{*}\right)$ and $y=T x$, then $y \in S^{\prime}$ with $Q y=x^{*}$ by Theorem $1(\mathrm{~d})$,

$$
\begin{aligned}
\left\|y-x_{0}\right\| & =\left\|x^{*}-x_{0}+H(I-P) N x-H(I-P) N x_{0}+\gamma\right\| \\
& \leqq c+k\left\|N x-N x_{0}\right\|+e \\
& \leqq d
\end{aligned}
$$

and similarly, $\mu\left(y-x_{0}\right) \leqq R_{0}$. Thus, $T$ maps $S\left(x^{*}\right)$ into itself. Note that $T$ is a contraction, and hence, by the Banach Fixed Point Theorem the auxiliary equation (7) is uniquely solvable in $S\left(x^{*}\right)$. Also, if $x^{*} \in V$ and $y^{*} \in V$, and if $x \in S\left(x^{*}\right)$ and $y \in S\left(y^{*}\right)$ are the unique elements with

$$
x=x^{*}+H(I-P) N x, \quad y=y^{*}+H(I-P) N y,
$$

then

$$
\begin{aligned}
\|x-y\| & \leqq\left\|x^{*}-y^{*}\right\|+\|H(I-P)(N x-N y)\| \\
& \leqq\left\|x^{*}-y^{*}\right\|+k l\|x-y\|,
\end{aligned}
$$

or $\|x-y\| \leqq(1-k l)^{-1}\left\|x^{*}-y^{*}\right\|$. This completes the proof of the theorem.

Theorem 2 guarantees that the auxiliary equation (7) can be solved for each $x^{*} \in V$. In fact, it permits us to set up a correspondence between $x^{*} \in V$ and the solution $x \in S\left(x^{*}\right)$ of the auxiliary equation: under the hypothesis of Theorem 2 let $\Gamma: V \rightarrow \mathscr{D}(L) \cap \tilde{S}_{0}$ be the continuous operator defined by $\Gamma\left(x^{*}\right)=x$ for $x^{*} \in V$ where $x$ is the unique element in $S\left(x^{*}\right)$ which is a solution to the auxiliary equation (7) corresponding to $x^{*}$.

Note that $\Gamma\left(x^{*}\right) \in \mathscr{D}(L) \cap \mathscr{D}(N)$ for each $x^{*} \in V$, and hence, $P\left(L \Gamma x^{*}-N \Gamma x^{*}\right)$ is an operator mapping $V$ into the subspace $\left\langle\omega_{1}, \ldots, \omega_{m}\right\rangle$. The next theorem is an immediate consequence of Theorem 2.

THEOREM 3. Let conditions (Iabc) and (IIabcd) be satisfied and let relations (13) be valid. If there exists an element $x^{*} \in V$ such that

$$
P\left(L \Gamma x^{*}-N \Gamma x^{*}\right)=0,
$$

then the element $\hat{x}=\Gamma x^{*}$ is a solution of the equation $L x=N x, Q \hat{x}=x^{*}$, and $\left\|\hat{x}-x_{0}\right\| \leqq d, \mu\left(\hat{x}-x_{0}\right) \leqq R_{0}$.

In Theorem 3 the problem of solving equation (1) has been reduced to the problem of solving equation (14), which is actually a system of $m$ equations in $p+m-q$ unknowns. Equation (14) is called the bifurcation equation or the determining equation. We examine it in the next section.

4. The bifurcation equation. In this section sufficient conditions are introduced for the existence of a solution $x^{*} \in V$ to the bifurcation equation (14). Let $\psi: \mathscr{D}(L) \cap \mathscr{D}(N) \rightarrow\left\langle\omega_{1}, \ldots, \omega_{m}\right\rangle$ be the operator defined by

$$
\psi x=P(L x-N x) \text { for all } x \in \mathscr{D}(L) \cap \mathscr{D}(N) .
$$


Note that if conditions (IIac) are satisfied, then $V$ and $\mathscr{D}(L) \cap \tilde{S}_{0}$ are both subsets of $\mathscr{D}(L) \cap \mathscr{D}(N)$, and for elements $x, y$ belonging to $\mathscr{D}(L) \cap \widetilde{S}_{0}$ :

$$
\begin{aligned}
\|\psi x-\psi y\| & =\left\|\sum_{i=1}^{m}\left(L x-L y, \omega_{i}\right) \omega_{i}-\sum_{i=1}^{m}\left(N x-N y, \omega_{i}\right) \omega_{i}\right\| \\
& \leqq \sum_{i=1}^{m}\left|\left(x-y, L^{*} \omega_{i}\right)\right|+\sum_{i=1}^{m}\left|\left(N x-N y, \omega_{i}\right)\right| \\
& \leqq\left[\sum_{i=q+1}^{m}\left\|L^{*} \omega_{i}\right\|+m l\right]\|x-y\| .
\end{aligned}
$$

Throughout the remainder of this section we assume that conditions (Iabc) and (IIabcd) are satisfied and that relations (13) are valid. Thus, the continuous operators $\Gamma, \psi \mid \mathscr{D}(L) \cap \tilde{S}_{0}$, and $\psi \mid V$ exist with

$$
V \stackrel{\Gamma}{\longrightarrow} \mathscr{D}(L) \cap \tilde{S}_{0} \stackrel{\psi}{\longrightarrow}\left\langle\omega_{1}, \ldots, \omega_{m}\right\rangle
$$

and

$$
V \stackrel{\psi}{\longrightarrow}\left\langle\omega_{1}, \ldots, \omega_{m}\right\rangle .
$$

Note that $\psi \Gamma$ maps the "ball" $V$, which is a subset of the $p+m-q$ dimensional space $S_{0}$, continuously into the $m$ dimensional space $\left\langle\omega_{1}, \ldots, \omega_{m}\right\rangle$, and also that the bifurcation equation (14) can be rewritten as

$$
\psi \Gamma x^{*}=0 .
$$

The operator $\psi \Gamma$ is difficult to work with because $\Gamma$ is defined by an iteration process. On the other hand, $\psi \mid V$ is easily obtained. The following lemma relates these two operators.

LEMMA. Let conditions (Iabc) and (IIabcd) be satisfied, and let relations (13) be valid. Then $\left\|\psi \Gamma x^{*}-\psi x^{*}\right\| \leqq(k l d+e)$ l for all $x^{*} \in V$.

Proof. Take $x^{*} \in V$ and let $x=\Gamma x^{*}$. Then $x \in S\left(x^{*}\right), Q x=Q x^{*}$, and $P L x=P L x^{*}$, so $\psi \Gamma x^{*}-\psi x^{*}=P\left(N x^{*}-N x\right)$. Hence, by Bessel's inequality and (IIc) we have

$$
\begin{aligned}
\left\|\psi \Gamma x^{*}-\psi x^{*}\right\| & \leqq l\left\|x-x^{*}\right\| \\
& \leqq l\left\|H(I-P) N x-H(I-P) N x_{0}+\gamma\right\| \\
& \leqq(k l d+e) l .
\end{aligned}
$$

We use this lemma to determine conditions on $\psi \mid V$ which guarantee that the bifurcation equation (14) is solvable.

Apply the Gram-Schmidt process to the elements $H \omega_{q+1}, \ldots, H \omega_{m}$ to obtain orthonormal elements $\eta_{q+1}, \ldots, \eta_{m}$. Let $M=p+m-q$, and let $E^{M}$ be a copy of Euclidean $M$-space where we represent each point $\xi \in E^{m}$ as an $M$-tuple: $\xi=\left(b_{1}, \ldots, b_{p}, c_{q+1}, \ldots, c_{m}\right)$. Also, let $E^{m}$ be a copy of Euclidean $m$-space where we 
represent each point $u \in E^{m}$ as an $m$-tuple: $u=\left(u_{1}, \ldots, u_{m}\right)$. We define two operators $\Gamma_{1}: E^{M} \rightarrow S_{0}$ and $\Gamma_{2}:\left\langle\omega_{1}, \ldots, \omega_{m}\right\rangle \rightarrow E^{m}$ by

$$
\Gamma_{1}\left(b_{1}, \ldots, b_{p}, c_{q+1}, \ldots, c_{m}\right)=\sum_{i=1}^{p} b_{i} \phi_{i}+\sum_{i=q+1}^{m} c_{i} \eta_{i}
$$

and

$$
\Gamma_{2}\left(\sum_{i=1}^{m} u_{i} \omega_{i}\right)=\left(u_{1}, \ldots, u_{m}\right)
$$

Clearly $\Gamma_{1}$ and $\Gamma_{2}$ are isomorphisms. Let $\xi_{0} \in E^{M}$ be the element with $\Gamma_{1}\left(\xi_{0}\right)=x_{0}$, and let $\Psi: E^{M} \rightarrow E^{m}$ be the operator

$$
\Psi=\Gamma_{2} \psi \Gamma_{1} .
$$

Choose a number $\varepsilon>0$ such that the set

$$
U=\left\{\xi \in E^{M} \mid\left\|\xi-\xi_{0}\right\| \leqq \varepsilon\right\}
$$

is mapped by $\Gamma_{1}$ into the set $V$. The existence of such an $\varepsilon$ is not difficult to show. We observe that the operators $\Gamma_{2} \psi \Gamma_{1}$ and $\Gamma_{2} \psi \Gamma \Gamma_{1}$ map the ball $U$, which is a subset of $E^{M}$, continuously into $E^{m}$. This is used in the next theorem to obtain an existence theorem for equation (1) for the case that $E^{m}$ has dimension 1 .

THEOREM 4. Let $m=1$, let conditions (Iabc) and (IIabcd) be satisfied, and let relations (13) be valid. If there exists a number $\delta>0$ such that the interval $[-\delta, \delta]$ is a subset of $\Psi(U)$ and if $(k l d+e) l \leqq \delta$, then there exists $x^{*} \in V$ such that the element $\hat{x}=\Gamma\left(x^{*}\right)$ is a solution of the equation $L x=N x$, and $Q \hat{x}=x^{*},\left\|\hat{x}-x_{0}\right\| \leqq d$, $\mu\left(\hat{x}-x_{0}\right) \leqq R_{0}$.

Proof. Choose $\xi_{1}, \xi_{2} \in U$ such that $\Psi\left(\xi_{1}\right)=\delta$ and $\Psi\left(\xi_{2}\right)=-\delta$. Let $x_{1}^{*}=\Gamma_{1}\left(\xi_{1}\right)$, $x_{2}^{*}=\Gamma_{1}\left(\xi_{2}\right)$. Clearly $x_{1}^{*}$ and $x_{2}^{*}$ are elements of $V$, and by the lemma we have $\left\|\psi \Gamma x_{i}^{*}-\psi x_{i}^{*}\right\| \leqq \delta$ for $i=1,2$. Thus,

$$
\begin{aligned}
\left|\Gamma_{2} \psi \Gamma \Gamma_{1}\left(\xi_{1}\right)-\delta\right| & =\left|\Gamma_{2} \psi \Gamma \Gamma_{1}\left(\xi_{1}\right)-\Gamma_{2} \psi \Gamma_{1}\left(\xi_{1}\right)\right| \\
& =\left\|\psi \Gamma x_{1}^{*}-\psi x_{1}^{*}\right\| \leqq \delta,
\end{aligned}
$$

or $\Gamma_{2} \psi \Gamma \Gamma_{1}\left(\xi_{1}\right) \geqq 0$. Similarly, $\Gamma_{2} \psi \Gamma \Gamma_{1}\left(\xi_{2}\right) \leqq 0$. Since $\Gamma_{2} \psi \Gamma \Gamma_{1}(U)$ is connected, there exists $\xi \in U$ such that $\Gamma_{2} \psi \Gamma \Gamma_{1}(\xi)=0$. If we set $x^{*}=\Gamma_{1}(\xi)$, then $x^{*} \in V$ and $P\left(L \Gamma x^{*}-N \Gamma x^{*}\right)=0$. The proof is completed using Theorem 3 .

To conclude this section we give another existence theorem which relaxes the condition $m=1$. Let conditions (Iabc) and (IIabcd) be satisfied, and let relations (13) be valid. In addition we assume that the following conditions are satisfied:

(IIIa) $p \geqq q$,

(IIIb) $\Psi\left(\xi_{0}\right)=0$,

(IIIc) The first order partial derivatives of $\Psi$ exist and are continuous on $U$,

(IIId) The Jacobian matrix for $\Psi$ has rank $m$ at $\xi_{0}$.

The first condition is equivalent to the condition $M \geqq m$, implying that $\Psi$ maps from a high dimensional space into a lower dimensional space. The second condition says that $P\left(L x_{0}-N x_{0}\right)=0$, which means that $x_{0}$ can be considered as an 
approximate solution to equation (1). In applications this suggests how one should choose $x_{0}$. The third condition corresponds to putting certain differentiability conditions on the operator $N$. The last condition guarantees that the range of $\Psi$ covers a neighborhood of the origin in $E^{m}$. In fact, by the Inverse Function Theorem we can choose a number $\delta>0$ such that the set

$$
W=\left\{u \in E^{m} \mid\|u\| \leqq \delta\right\}
$$

is a subset of $\Psi(U)$, and such that there exists a continuous mapping $\Lambda: W \rightarrow U$ with $\Psi[\Lambda(u)]=u$ for all $u \in W$.

THEOREM 5. Let conditions (Iabc), (IIabcd), and (IIIabcd) be satisfied, and let relations (13) be valid. If $\delta>0$ is a number chosen as above and if $(k l d+e) l<\delta$, then there exists $x^{*} \in V$ such that the element $\hat{x}=\Gamma\left(x^{*}\right)$ is a solution of the equation $L x=N x$, and $Q \hat{x}=x^{*},\left\|\hat{x}-x_{0}\right\| \leqq d, \mu\left(\hat{x}-x_{0}\right) \leqq R_{0}$.

Proof. Consider the two continuous maps $\Gamma_{2} \psi \Gamma \Gamma_{1} \Lambda: W \rightarrow E^{m}$ and $I: W \rightarrow E^{m}$ where $I(u) \equiv u$. Take $u \in W$ and let $x^{*}=\Gamma_{1} \Lambda(u)$. Then $x^{*} \in V, \Gamma_{2} \psi\left(x^{*}\right)=I(u)$, and

$$
\left\|\Gamma_{2} \psi \Gamma \Gamma_{1} \Lambda(u)-I(u)\right\|=\left\|\psi \Gamma\left(x^{*}\right)-\psi\left(x^{*}\right)\right\| \leqq(k l d+e) l,
$$

or

$$
\left\|\Gamma_{2} \psi \Gamma \Gamma_{1} \Lambda(u)-I(u)\right\|<\delta \text { for all } u \in W .
$$

This inequality implies that for each $u \in \partial W$, the line segment joining $I(u)$ and $\Gamma_{2} \psi \Gamma \Gamma_{1} \Lambda(u)$ does not contain the origin of $E^{m}$. By the Poincaré-Bohl Theorem [6, p. 32] the local degree of $\Gamma_{2} \psi \Gamma \Gamma_{1} \Lambda$ at 0 relative to $W$ is equal to the local degree of $I$ at 0 relative to $W: d\left(\Gamma_{2} \psi \Gamma \Gamma_{1} \Lambda, W, 0\right)=d(I, W, 0)$. But $d(I, W, 0)=1$, and hence, $d\left(\Gamma_{2} \psi \Gamma \Gamma_{1} \Lambda, W, 0\right) \neq 0$. Therefore, there exists $u \in W$ such that $\Gamma_{2} \psi \Gamma \Gamma_{1} \Lambda(u)=0$. Setting $x^{*}=\Gamma_{1} \Lambda(u)$, we have $x^{*} \in V$ and $P\left(L \Gamma x^{*}-N \Gamma x^{*}\right)=0$. The proof is completed using Theorem 3 .

5. An application. We illustrate our existence theory by studying the nonlinear boundary value problem:

$$
\begin{aligned}
x^{\prime \prime}(t)+x(t)+\alpha x^{2}(t) & =\beta t, \quad 0 \leqq t \leqq 2 \pi, \\
x(0) & =0,
\end{aligned}
$$

where $\alpha$ and $\beta$ are real constants. Let $I$ be the interval $[0,2 \pi]$, let $S$ be the real Hilbert space $L_{2}(I)$, let $S^{\prime}$ be the subspace in $S$ consisting of all functions which are bounded a.e., and let $\mu$ be the uniform norm in $S^{\prime}$, i.e., $\mu(x)=\inf \{c|| x \mid \leqq c$ a.e. $\}$.

We denote by $H^{2}(I)$ the subspace of $S$ consisting of all functions $x(t)$ with the properties: $x$ is continuous on $I, x^{\prime}$ exists and is absolutely continuous on $I, x^{\prime \prime} \in S$. Let $L$ be the 2 nd order differential operator in $S$ defined by $L x=x^{\prime \prime}+x$ where the domain $\mathscr{D}(L)$ consists of all functions $x \in H^{2}(I)$ with $x(0)=0$. It is well known [13, pp. 431-434] that $L$ is a closed linear operator in $S$ with dense domain and closed range, and that the adjoint $L^{*}$ is the 2nd order differential operator in $S$ given by $L^{*} x=x^{\prime \prime}+x$ where $\mathscr{Z}\left(L^{*}\right)$ consists of all functions $x \in H^{2}(I)$ with $x(0)=x(2 \pi)=0, x^{\prime}(2 \pi)=0$. Since $\mathscr{N}(L)$ and $\mathscr{N}\left(L^{*}\right)$ are subsets of $\langle\sin t, \cos t\rangle$, 
both null spaces are finite-dimensional. In fact, it is easy to check that $\mathscr{N}(L)=\langle\sin t\rangle$ and $\mathscr{N}\left(L^{*}\right)=\langle 0\rangle$. Thus, $L$ is a closed linear operator in $S$ with properties (Iabc), $p=1, q=0$, and $\mathscr{R}(L)=S$.

Let $N$ be the operator in $S$ defined by $\mathscr{D}(N)=S^{\prime}, N x=-\alpha x^{2}(t)+\beta t$ for all $x(t) \in \mathscr{D}(N)$. Note that $\mathscr{D}(L) \cap \mathscr{D}(N)=\mathscr{D}(L)$, which is a subset of $S^{\prime}$. The equation $L x=N x$ is equivalent to the nonlinear boundary value problem (21). Using Theorem 4, we show that there exists a solution $\hat{x}$ to the equation $L x=N x$ for all $(\alpha, \beta)$ with $|\alpha| \leqq 1,|\beta| \leqq .001$.

Choose $\phi_{1}(t)=\pi^{-1 / 2} \sin t$, and let $G(t, s)=\sin t \cos s-\cos t \sin s$. Then the right inverse operator $H$ can be shown to have the integral representation

$$
H y(t)=\int_{0}^{t} G(t, s) y(s) d s+\sin t \cdot \int_{0}^{2 \pi} g(s) y(s) d s, \quad 0 \leqq t \leqq 2 \pi,
$$

for all $y \in S$, where $g(t)=-\cos t+(t / 2 \pi) \cos t-(1 / 2 \pi) \sin t$.

Next, observe that the function $\omega(t)=(t-2 \pi) \sin t$ belongs to $\mathscr{D}\left(L^{*}\right)$ : choose $m=1$ and $\omega_{1}(t)=\|\omega\|^{-1} \omega(t)$. For this choice of $m$ and $\omega_{1}, M=p+m-q=2$ and the operators $P, Q, \psi, \Gamma_{1}, \Gamma_{2}$, and $\Psi$ are given by (5), (6), (15), (16), (17), and (18), respectively. In particular, $\Psi: E^{2} \rightarrow E^{1}$ is given by

$$
\Psi\left(b_{1}, c_{1}\right)=.502738 c_{1}-.211427 \alpha b_{1}^{2}+.093851 \alpha b_{1} c_{1}+.033884 \alpha c_{1}^{2} .
$$

Also, the subspace $S_{0}=\left\langle\phi_{1}, H \omega_{1}\right\rangle$ has dimension 2 and is a subset of $\mathscr{D}(N)$.

The element $x_{0} \in S_{0}$ is chosen so that $\psi\left(x_{0}\right)=0$ or $\Psi\left(\xi_{0}\right)=0$. From (23) we note that the latter condition is satisfied for $\xi_{0}=(0,0)$, and this choice of $\xi_{0}$ yields $x_{0}(t) \equiv 0$. Then $\gamma=H(I-P) N x_{0}$ is given by $\gamma(t)=2 \beta \sin t+\beta t$ with $\|\gamma\|=8.373592|\beta|$ and $\mu(\gamma)=2 \pi|\beta|$. Let $e=8.374|\beta|$ and $e^{\prime}=6.284|\beta|$.

It is clear that condition (IIa) is satisfied. By means of (22) the operator $H(I-P)$ can also be shown to be an integral operator of the form

$$
H(I-P) x(t)=\int_{0}^{2 \pi} K_{1}(t, s) x(s) d s, \quad 0 \leqq t \leqq 2 \pi,
$$

for all $x \in S$. Thus, condition (IIb) is satisfied provided

and

$$
k \geqq\left(\int_{0}^{2 \pi} \int_{0}^{2 \pi}\left[K_{1}(t, s)\right]^{2} d s d t\right)^{1 / 2}=1.413573
$$

$$
k^{\prime} \geqq\left(\max _{0 \leqq t \leqq 2 \pi} \int_{0}^{2 \pi}\left[K_{1}(t, \xi)\right]^{2} d \xi\right)^{1 / 2}=.832194 .
$$

Let $k=1.414$ and $k^{\prime}=.833$. For condition (IIc) the set $\tilde{S}_{0}$ is given by

$$
\tilde{S}_{0}=\left\{x \in S^{\prime} \mid\|x\| \leqq d, \mu(x) \leqq R_{0}\right\},
$$

which is a subset of $\mathscr{D}(N)$, and for $x \in \tilde{S}_{0}, y \in \tilde{S}_{0}$ we have

$$
\begin{aligned}
|N x(t)-N y(t)| & =|\alpha\|y(t)+x(t)\| y(t)-x(t)| \\
& \leqq 2 R_{0}|\alpha||x(t)-y(t)|
\end{aligned}
$$

and $\|N x-N y\| \leqq 2 R_{0}|\alpha|\|x-y\|$. Let $l=2 R_{0}|\alpha|$. Condition (IId) has been shown to hold by Cesari [3, p. 404]. Thus, conditions (IIabcd) are satisfied for these choices of $k, k^{\prime}$, and $l$. 
For any function $x(t) \in S_{0}$ with $\|x\| \leqq c$, we can show that $|x(t)| \leqq 3.049797 c$. Hence, setting $r=3.049797 c$ and $\varepsilon=c$, the sets $V$ and $U$ simplify to

$$
V=\left\{x \in S_{0} \mid\|x\| \leqq c\right\} \text { and } U=\left\{\xi \in E^{2} \mid\|\xi\| \leqq c\right\} .
$$

If we assume that $|\alpha| \leqq 1$ and set $\delta=.502738 c-.033884 c^{2}$, then from (23) we obtain $\Psi(0, c) \geqq \delta$ and $\Psi(0,-c) \leqq-\delta$; under these assumptions the interval $[-\delta, \delta]$ is a subset of $\Psi(U)$.

Finally, to apply Theorem 4, we need to determine a bound on the parameter $\beta$ and choose the numbers $c, d$, and $R_{0}$ such that relations (13) are valid and such that $(k l d+e) l \leqq \delta$ :

$$
\begin{aligned}
& 0<c<d, \quad 3.049797 c<R_{0}, \\
& (1.414)\left(2 R_{0}\right)<1, \\
& c+8.374|\beta|+(1.414)\left(2 R_{0}\right) d \leqq d, \\
& 3.049797 c+6.284|\beta|+(.833)\left(2 R_{0}\right) d \leqq R_{0}, \\
& {\left[(1.414)\left(2 R_{0}\right) d+8.374|\beta|\right]\left(2 R_{0}\right) \leqq .502738 c-.033884 c^{2} .}
\end{aligned}
$$

One solution of these inequalities is given by $|\alpha| \leqq 1,|\beta| \leqq .001, c=.01, d=.03$, and $R_{0}=.1$, which yields $r=.030498, l=.2|\alpha|, \varepsilon=.01$, and $\delta=.005024$. From Theorem 4 we conclude that for each pair of real numbers $(\alpha, \beta)$ with $|\alpha| \leqq 1,|\beta| \leqq .001$ there exists a real-valued function $x(t)$ which is twice continuously differentiable on the interval $[0,2 \pi]$, and which is a solution of equation (21) with $\|x\| \leqq .03$ and $|x(t)| \leqq .1$.

\section{REFERENCES}

1. N. I. Ahiezer and L. M. Glazman, Theory of linear operators in Hilbert space. I, II, Ungar, New York, 1961.

2. R. G. Bartle, Singular points of functional equations, Trans. Amer. Math. Soc. 75 (1953), 336-384.

3. L. Cesari, Functional analysis and Galerkin's method, Michigan Math. J. 11 (1964), 384-414.

4. - - A nonlinear problem in potential theory, Report No. 2, NSF Research Project GP-57.

5. -__-, Functional analysis and periodic solutions of nonlinear differential equations, Contributions to Differential Equations 1 (1963), 149-187.

6. J. Cronin, Fixed points and topological degree in nonlinear analysis, Math. Surveys, No. 11, Amer. Math. Soc., Providence, R. I., 1964.

7. - - Branch points of solutions of equations in Banach space, Trans. Amer. Math. Soc. 69 (1950), 208-231.

8. N. Dunford and J. T. Schwartz, Linear operators. I, II, Interscience, New York, 1958.

9. A. Ljapunov, Sur les figures d'équilibre peu différentes des ellipsoides d'une masse liquide homogène douée d'un mouvement de rotation. Première Partie. Étude générale du problème, Mémoire présenté à l'Académie Impériale des Sciences, March 21, 1906, St. Petersbourg, 1-225.

10. L. Nirenberg, Functional analysis, New York University Lectures, 1960-1961. 
11. H. Poincaré, Les méthodes nouvelles de la mécanique céleste, Vols. 1, 2, 3, GauthierVillars, Paris, 1892-1899; reprinted by Dover, New York, 1957.

12. E. Schmidt, Zur Theorie der linearen und nichtlinearen Integralgleichungen, Math. Ann. 65 (1907-1908), 370-399.

13. J. Schwartz, Perturbations of spectral operators, and applications. I, Pacific J. Math. 4 (1954), 415-458.

UNIVERSITY OF MICHIGAN,

ANN ARbor, Michigan 